\title{
A Single Resolved Ground-Based Observation of Mercury
}

\author{
Leonid V. Ksanfomality \\ Space Research Institute, Moscow, Russia
}

\begin{abstract}
Short-exposure images of Mercury were recorded at the Abastumany astrophysical observatory. A single image, with low distortion, is shown and discussed with respect to Mariner 10 observations.
\end{abstract}

\section{Introduction}

The results of investigations of astronomical climate in several observatories have shown that the amplitudes of scintillation (flicker) decrease for stars with higher frequency spectra (Ksanfomality \& Shakhovskoy 1967). This means that a short exposure may substantially improve the image resolution of planetary astronomical objects. The use of a high sensitivity CCD detector permits the exposure to be radically diminished. This improves the sharpness of the image, however it does not improve picture distortion. Nevertheless it is possible to find a low distortion picture amid many obtained in observations.

\section{Observations}

Fifteen short exposure $(10 \mathrm{~ms})$ images of the planet Mercury were obtained in December 1999 , with a zenith angle of $83-78^{\circ}$, at the Abastumany astrophysical observatory (Republic of Georgia), located at an altitude of $1700 \mathrm{~m}$ a.s.l. Only one image $(\lambda=533 \mathrm{~nm}, \Delta \lambda=24 \mathrm{~nm})$ is of low distortion. The telescope was an AZT-11 $(\mathrm{D}=1.25 \mathrm{~m}, \mathrm{~F}=16 \mathrm{~m})$. Mercury was at a geocentric distance of 1.007 $\mathrm{AU}$, a heliocentric distance of $0.39 \mathrm{AU}$ and had a phase of $76^{\circ}$. The coordinates of the subterrestrial point at 03:30 UT, 3.12 .1999 were $108^{\circ} \mathrm{W}, 2^{\circ} 26^{\prime}$. The CCD pixel size was $12 \times 14 \mu \mathrm{m}$. The photometric readings were about 1000 counts per pixel.

All images are additionally distorted by refraction in their eastern parts. Despite the fact that the observations were made far from the zenith, about $80^{\circ}$, photometrical sections along the subterrestrial meridian show some details seen in the curve (Fig. 1).

The suggestion was made that the details seen in the image reveal large blocks of Hermean surface, normally not seen in ground based images of Mercury (Vilas, Chapman, \& Matthews 1988). Occasionally the phase of the planet and the subterrestrial point position were almost the same as when Mariner 10 encountered Mercury in 1974. This coincidence permits a comparison of the images obtained with the Mariner 10 data, proving the reality of the details. 


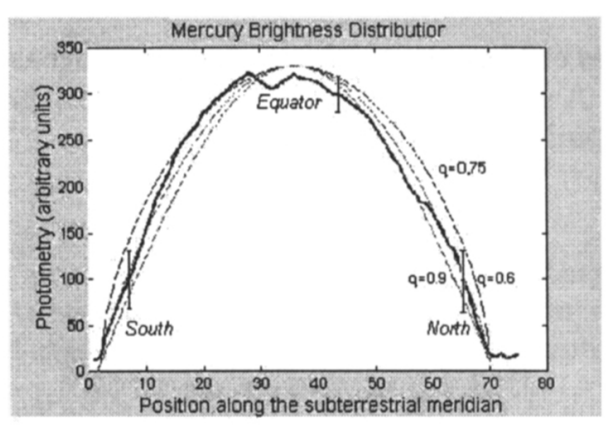

Figure 1. Comparison of the Mercury photometry along the subterrestrial meridian and a $(\cos \phi)^{q}$ model for the $76^{\circ}$ phase.
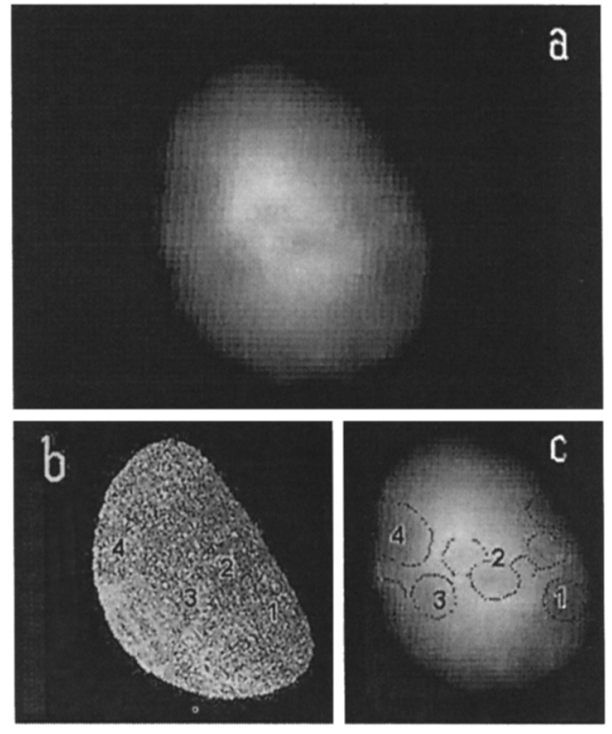

Figure 2. Positions of large details on Mercury's disk (a) in comparison with a MARINER 10 image of the planet (b) and a scheme of large blocks (c).

The large dark area 1 was identified with crater Beethoven $\left(20^{\circ} \mathrm{S}, 124^{\circ} \mathrm{W}\right.$, center). The three dark spots 2 in the disk center and $120-140^{\circ} \mathrm{W}$ are also seen in section (b), as is spot 3. Spot 4 was seen in section (b).

\section{Conclusion}

Using very short exposures for Mercury ground-based observations allows resolved images of the planet to be obtained that are not available using any other ground-based techniques. The image resolution is up to $0.4^{\prime \prime}$ instead of 1 to $1.5^{\prime \prime}$ for a moderate sized telescope. The method has much in common with speckle interferometry.

\section{References}

Ksanfomality, L. V., \& Shakhovskoy, A. M. 1967, Proc. Crimean Astrophys. Observ., 38,264

Vilas, F., Chapman, C.R., \& Matthews, M.S. (eds.) 1988, Mercury (Tucson: Univ. Arizona Press) 